\title{
OBSERVATORIO
}

\section{SOPORTES DIGITALES Y TRANSFORMACIÓN DE LAS INDUSTRIAS DE CONTENIDOS}

\section{Claudio Feijóo}

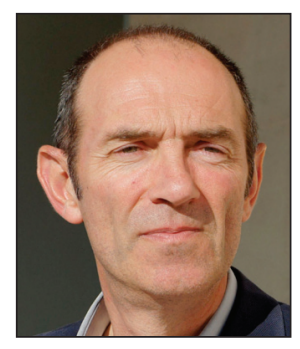

Claudio Feijóo, doctor ingeniero de telecomunicación y licenciado en economía, es subdirector del Centro de Investigación sobre Edificios Inteligentes y Eficiencia Energética de la Universidad Politécnica de Madrid, donde se dedica a investigar las tecnologías emergentes de la sociedad de la información y su impacto socio-económico, en particular en todo lo relacionado con las comunicaciones móviles, la industria de los contenidos y las infraestructuras de nueva generación. http://orcid.org/0000-0002-9499-7790

Cedint-Universidad Politécnica de Madrid Campus de Montegancedo, Pozuelo de Alarcón, 28223 Madrid, Spain cfeijoo@cedint.upm.es

\section{Resumen}

Entre los efectos que la digitalización de las industrias de contenidos está teniendo, ocupa un lugar prominente la aparición de múltiples soportes y formatos inexistentes anteriormente. De hecho, son estos soportes y formatos los que los usuarios-consumidores perciben inmediatamente y, por tanto, son los que están transformando la relación del usuario con la información y, en particular, su capacidad de interacción con la misma. Sin embargo, y a pesar de esta considerable importancia, la relación bidireccional de los soportes y formatos con los usuarios ha sido poco estudiada. Precisamente este breve artículo trata de ofrecer algunas ideas generales sobre dónde aparecen los soportes y formatos en el marco general de la digitalización y hace dónde se dirige su evolución. Lo hace considerando algunos subsectores concretos del ámbito de los contenidos como son la edición y distribución de libros y periódicos.

\section{Palabras clave}

Industria de los contenidos, Soportes digitales, Formatos digitales, Medios de comunicación, Sector editorial, Libros, Periódicos.

\section{Title: Digital media and the transformation of content industries}

\begin{abstract}
In media and content industries, the main effect of digitization has been a radical transformation of information formats and displays. In fact, these formats and displays are responsible for the shifting perceptions of users-consumers concerning how they relate and interact with information. However, in spite of its considerable relevance, the bidirectional relationship between formats/displays and users has been seldom studied. This brief paper presents an overview of the evolution of displays and devices in some key media and content subsectors -publishing and distribution of books and newspapers- with the main objective of hinting at the next steps in their evolution.
\end{abstract}

\section{Keywords}

Content industry, Digital media, Digital formats, Media, Publishing, Books, Newspapers.

Feijóo, Claudio (2013). "Soportes digitales y transformación de las industrias de contenidos". El profesional de la información, enero-febrero, v. 22, n. 1, pp. 5-9.

\section{El marco de la digitalización ${ }^{1}$}

Hasta hace tan solo dos décadas, las denominadas industrias culturales ${ }^{2}$ eran un ámbito relativamente maduro, caracterizado desde el punto de vista económico fundamentalmente por considerables economías de escala ${ }^{3}$ y alcance. Los costes de producir una "primera copia" eran altos, mien- tras que los correspondientes a las siguientes copias -los soportes ${ }^{4}$ - eran notoriamente menores. Como consecuencia la estructura de la industria presentaba elevadas barreras a la entrada y tendía hacia la concentración empresarial estructura oligopolística. Además, los mercados estaban segmentados culturalmente, con notables ventajas para las compañías basadas en amplias comunidades homogéneas, 
creando una distribución "natural" geográfica de actividades empresariales. Desde el punto de vista de lo público, la existencia de externalidades positivas, es decir, de beneficios para el conjunto de la sociedad más allá del valor individual de la provisión de contenidos, se ha citado tradicionalmente como el principal argumento para diversos tipos de intervenciones regulatorias. Estas intervenciones van desde la fijación de condiciones para que el pluralismo cultural quede asegurado hasta la provisión directa de contenidos e información a través de diversas variantes del concepto de servicio público, es decir, como provisión directa por parte de las administraciones públicas, a través de entes públicos empresariales o mediante gestión privada de la titularidad pública del servicio.

En este marco la tecnología ha traído -está trayendo- cambios radicales a través del fenómeno de la digitalización. En primer lugar, la digitalización ha disminuido enormemente los costes de distribución y considerablemente los costes de producción. Además, la tecnología ha facilitado que se produzca un cambio socio-económico en los consumidores, que se manifiesta fundamentalmente en una mayor propensión a la privatización -individualización y apropiación en la esfera de lo privado- del consumo de contenidos e información; en una mayor facilidad en el manejo de diferentes y nuevos medios; en un desplazamiento en la valoración relativa de cada uno de estos diferentes medios hacia los más actuales en detrimento de los tradicionales; en una creciente interacción con la información; y en su participación, siquiera parcial, en la creación de contenidos e información. Los consumidores están ahora más conectados que nunca, son más interactivos, más móviles, más personales y más sociales.

Los consumidores están ahora más conectados que nunca, son más interactivos, más móviles, más personales y más sociales

Sin embargo, antes de proseguir conviene recordar que la digitalización ha cambiado esta industria pero algunas características de la misma se mantienen: las economías de escala y alcance, las externalidades y la segmentación cultural. De hecho, aunque inicialmente se ha producido un fenómeno de desintermediación resultado de la bajada de las barreras a la entrada de nuevos agentes, hemos comenzado a experimentar ya un nuevo proceso de "re-intermediación" liderado por nuevos agentes digitales como más adelante se explica. En cualquier caso, la digitalización ha traído un sinnúmero de innovaciones en aplicaciones, servicios, soportes y modelos de negocio, cambiando la propia estructura de las industrias existentes y, desde luego, planteando nuevos retos hasta ahora desconocidos.

De entre todas estas innovaciones son los formatos y los soportes las menos estudiadas a pesar de su más que evidente influencia en la evolución del sector. Como ejemplo principal, todos los nuevos entrantes así como los nuevos medios que han aparecido en esta industria, lo han hecho apoyados en algún tipo de innovación en forma de soporte o formato digital. Los ejemplos son numerosos: teléfonos inteligentes, tablets, videoconsolas portátiles, televisores "conectados", libros electrónicos y un largo etcétera

\section{De los soportes digitales a las plataformas y viceversa}

Los efectos de los soportes digitales en la industria de los contenidos se pueden dividir en aquellos sobre la industria tradicional y los que se refieren al nuevo entorno digital. Con respecto a los primeros los efectos más destacados son consecuencia de la facilidad para hacer nuevas copias de diversos tipos de contenidos y distribuirlas. Así, la industria tradicional se ha enfrentado al hecho de que la piratería online ha impedido una transición "suave" hacia el nuevo entorno digital, sin olvidar, al mismo tiempo, que esta misma industria -miope y conservadora- no ha sabido probablemente aprovechar a tiempo las oportunidades de las innovaciones digitales -los soportes y formatos, en particular- para suministrar a los consumidores nuevas y atractivas soluciones que compensaran los efectos de esta piratería.

También hay que señalar que el éxito de los soportes digitales no significa necesariamente el fin de la industria tradicional, ni siquiera la desaparición de los formatos clásicos, simplemente el tamaño de esta industria será comparativamente menor. La industria fonográfica es el ejemplo más evidente. En su versión actual posee un tamaño de alrededor de la mitad de lo que alcanzó en su época de mayor esplendor a pesar de haber ampliado su radio de acción desde la mera venta de soportes físicos hasta el management y el merchandising de los artistas. Al mismo tiempo, la desvalorización del soporte digital físico -los cds- frente al consumo online ha hecho que formatos tradicionales como los discos de vinilo vuelvan a aparecer como un nicho limitado pero atractivo de mercado: coleccionistas, objeto artístico, etc.

En el caso de los nuevos medios digitales, además de los ya citados -reducción de costes en producción y distribución, y crecientes posibilidades para la interacción y personalización de la experiencia de usuario-, facilitan la provisión de servicios a distancia y con coste reducido, creando muchos incentivos para la "des-territorialización" de estas industrias $y$, en consecuencia, aumentando los efectos de las economías de escala y haciendo muy difícil el enfoque tradicional de la regulación.

Asimismo, la capacidad de personalización que ofrecen estos formatos permite al menos teóricamente una mayor y mejor segmentación de los consumidores -la "larga cola" de oportunidades de negocio para pequeños nichos de mercado-, y por tanto un mejor uso del catálogo de los proveedores. Los formatos digitales han permitido igualmente que aparezcan contenidos no-profesionales creados por los propios consumidores -los prosumers ${ }^{5}$ y compartirlos fácilmente en redes sociales en las que los usuarios pueden expresarse más libre y fácilmente que nunca, otra vez modificando las relaciones existentes en la industria tradicional y creando nuevos retos para la regulación. También los nuevos soportes permiten que el usuario tenga experiencias "transmediáticas", simultaneando el uso de diferentes dispositivos o cambiando de un dispositivo a otro para utilizar el mismo contenido o extensiones del mismo. Abun- 
dando en el tema, estos mismos soportes permiten que los usuarios adquieran una mayor libertad en el consumo de contenidos, originando innovaciones y modelos de negocio diferentes -time-shifting ${ }^{6}$ en el ámbito audiovisual, redes sociales basadas en contenidos o aplicaciones móviles, por citar algunos de los principales ejemplos.

Otra cuestión muy interesante es que para muchos de los nuevos agentes los contenidos no son más que un medio y no un fin en sí mismos -como podía ser para la industria tradicional-, ya que sus modelos de negocio (venta de hardware, publicidad...) requieren una "comoditización" de los contenidos para conseguir una masa crítica de consumidores. Asistimos entonces a una desvalorización al menos parcial de los contenidos para conseguir un mayor atractivo de los soportes digitales.

La consecuencia de todo ello es la creación de nuevas estructuras de valor, las plataformas, y de todo un ecosistema alrededor de las mismas. Las plataformas son una versión sui generis de los mercados multilaterales, donde los propietarios de la plataforma intentan atraer a todos los interesados: productores de contenidos e información, desarrolladores de aplicaciones, fabricantes de soportes, anunciantes $y$, por supuesto, consumidores. Aunque nuevas, estas estructuras siguen la estrategia tradicional de intentar adquirir una posición preponderante en cada uno de los correspondientes mercados. Precisamente son los soportes digitales una de las herramientas hoy obligatorias para el éxito de la plataforma y su correspondiente ecosistema. Las comunicaciones móviles son sin duda el mejor y mayor ejemplo de esta estrategia, pero también lo son los libros digitales, las tablets o la misma "computación en la nube" como ejemplo de "soporte sin soporte".

\section{Asistimos a una desvalorización de los contenidos para conseguir un mayor atractivo de los soportes digitales}

\section{Dos casos particulares: los sectores editoriales de libros y periódicos}

\section{Libros}

La industria editorial, en el caso de los libros, se puede decir que ha sido el último de los sectores de la industria de los contenidos en ser digitalizada7. Este detalle debería dar a esta industria la oportunidad de aprender de otros sectores que han pasado por el proceso de digitalización con anterioridad y en particular del uso de los soportes y formatos estratégicamente. Sin embargo, es un proceso aún en sus fases iniciales. Todavía en 2012 el mercado de los "ebooks" representaba únicamente el 4,5\% de las ventas de libros en los países desarrollados ${ }^{8}$. Aun así, la llegada de los libros electrónicos ha alterado la estructura de costes de la industria, igual que ha sucedido en otros sectores. Algunos han desaparecido (impresión, transporte físico), otros apenas han cambiado (remuneración de la creación, corrección de pruebas, posibles traducciones, proceso editorial), otros han experimentado considerables cambios (marketing y

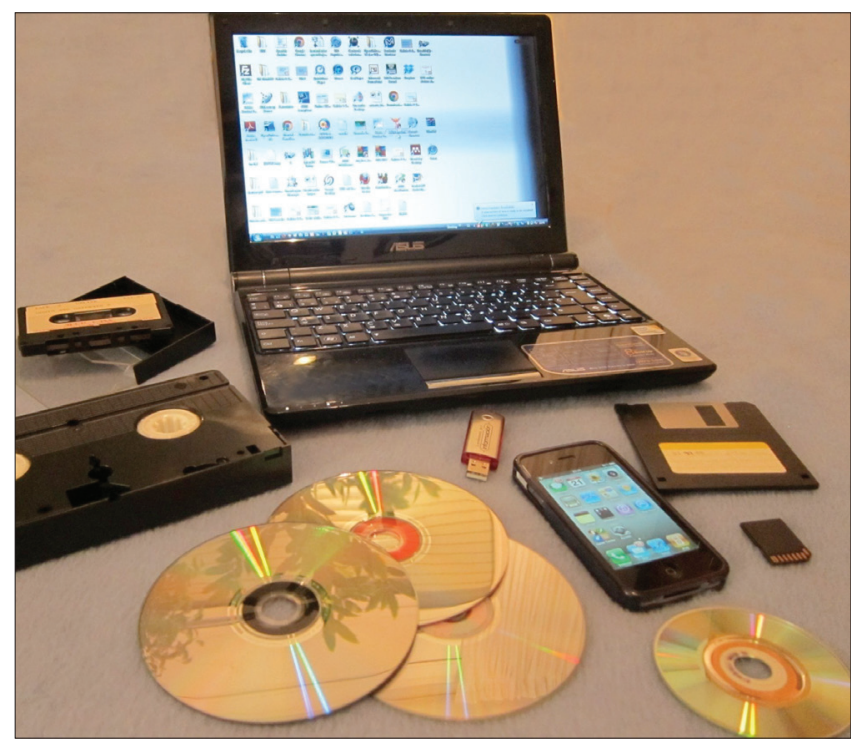

Soportes digitales

ventas), e incluso han aparecido costes nuevos ligados a la plataforma y los formatos y soportes necesarios. Se estima que los costes totales de los sistemas editoriales online son entre un 15 y un $25 \%$ más baratos que los tradicionales.

Desde el punto de vista europeo este sector tiene también la peculiaridad de ser el único, dentro de la industria de los contenidos, donde las compañías europeas tienen una posición de liderazgo. Sin embargo, y ésta es una cuestión verdaderamente preocupante, la transición a la era digital está liderada por compañías estadounidenses, que hacen uso intensivo de diversos soportes -dispositivos- para cimentar este liderazgo. De hecho la distribución online de libros está dominada por compañías ajenas a la industria editorial tradicional, y únicamente de forma muy reciente esta última ha reaccionado lanzando iniciativas para posicionar sus propias plataformas digitales y, por supuesto, sus propios dispositivos lectores. En cualquier caso, el mercado de los libros electrónicos es hoy simplemente una versión digital de su contraparte en papel con los mismos modelos de negocio.

El elemento clave para el desarrollo del mercado del libro electrónico son los soportes: lectores de libros electrónicos y tablets. Ahora mismo coexisten dos modelos muy diferentes: (i) modelos cerrados, ligados a dispositivos concretos, en los que el usuario únicamente puede adquirir los libros en la tienda del fabricante del dispositivo y no puede acceder a los mismos desde ningún otro, y (ii) modelos abiertos, que están basados en plataformas online accesibles desde cualquier dispositivo. La competencia entre estos dos modelos, que se repite en otros sectores de la industria de los contenidos, es uno de los rasgos característicos del actual modelo de plataformas digitales.

\section{Periódicos}

La edición de periódicos guarda parecido con la de libros pero tiene aspectos diferenciales de interés. Para empezar se trata de un sector que ha visto un fuerte descenso en la cantidad de ejemplares en circulación y una caída igualmente significativa en sus ingresos, todo ello, por supuesto 
derivado de la digitalización y del consumo de información periodística sobre otros formatos y soportes, distintos del tradicional papel. De hecho, los consumidores ya consideran a internet como la primera fuente de información, al menos en cuanto a inmediatez, si no en cuanto a fiabilidad.

Lo paradójico del caso, es que al mismo tiempo que se produce este declive, la demanda de información online no deja de crecer, así como el número y tipo de formatos y modelos de producción y diseminación de noticias. De hecho, el periodismo online ha sido citado numerosas veces como la actividad que compensaría este declive de la industria tradicional, dejando a un lado que su estructura, las habilidades requeridas para ejercerlo, o incluso sus valores, son bastante diferentes a los del modelo convencional. En realidad el modelo de negocio derivado de comprar y leer un periódico es bastante distinto de leer la misma información gratuitamente online y, desde luego la publicidad que se consigue mediante este último modelo no está generando ingresos suficientes como para compensar los ingresos tradicionales. Hasta ahora los periódicos en su versión digital han intentado replicar mayoritariamente los modelos de negocio de la versión impresa, incluyendo el pago por ejemplar, la suscripción, y la publicidad en diversas combinaciones y, salvo alguna excepción limitada, con resultados no demasiado halagüeños. Dentro de este panorama, otro modelo como el de agregación de noticias procedentes de diferentes fuentes facilitado por internet genera una considerable controversia. Desde el punto de vista de la industria editorial estos servicios digitales de agregación hacen uso -y se benefician económicamente- de las noticias publicadas por otros sin pagar por ellas.

Es por todo esto que la aparición de soportes digitales como tablets o lectores electrónicos ha traído un rayo de esperanza a la industria editorial. Varios periódicos y revistas han lanzado aplicaciones específicas para estos dispositivos con modelos típicamente de suscripción o "freemium", pero es pronto para saber si tienen un éxito destacable y sobre todo para conocer su sostenibilidad económica.

La aparición de tablets y lectores electrónicos ha traído un rayo de esperanza a la industria editorial

\section{Algunas conclusiones y un reto}

La digitalización de las industrias de los contenidos está en una fase inicial como muestran los indicadores de desarrollo de mercado y, por tanto, aún queda un largo camino de transformaciones pendientes. Los modelos de negocio son quizá el ejemplo más palmario. Tradicionalmente los ingresos venían de tres fuentes principales: ventas directas a los consumidores, suscripciones y publicidad. Sin embargo, todos y cada uno de estos tipos de ingresos se enfrentan a considerables retos en el ámbito digital. Comenzando por la publicidad, los anunciantes están trasladando sus presupuestos a las redes sociales y los buscadores, donde debido a su capacidad de personalización, piensan que es más fácil alcanzar a un público disperso. Los modelos de pago digita- les, aunque mucho más flexibles y capaces de adaptarse a las preferencias precisas de los consumidores, son todavía muy incipientes. Sin embargo, y al mismo tiempo, se atisba que sustituirán a la tradicional compra física en una muy amplia mayoría de casos y que las soluciones en la nube, donde el usuario acumula sus derechos a utilizar los contenidos desde diferentes tipos de dispositivos, tendrán un papel muy relevante.

Esta última es probablemente la conclusión más interesante desde el punto de vista de los formatos y soportes digitales. Nos encontramos ante una aparente, por así decirlo, esquizofrenia digital. Por un lado, los dispositivos que utilizan los consumidores de contenidos son cada vez más relevantes para estructurar la oferta de contenidos y organizar los modos de consumo. Son, como se ha visto, el elemento crítico de "enganche" de los usuarios de las diferentes plataformas que compiten en el mercado. Al mismo tiempo, los contenidos existentes se desmaterializan y se transforman en servicios online, a los que los usuarios quieren acceder de manera ubicua y sin importar el formato o el soporte que tengan en ese momento. Así, los usuarios están dejando de acumular obras físicas, al mismo tiempo que se rodean de todo tipo de terminales y multiplican los proveedores a los que acuden para hacer uso de los contenidos y acceder a la información.

Los usuarios dejan de acumular obras físicas, se rodean de todo tipo de terminales y multiplican los proveedores

Por ello, el principal reto que desde la perspectiva de los formatos y soportes digitales está pendiente de solución, es ofrecer al usuario una experiencia atractiva a través de diferentes medios y sistemas. Es lo que se viene a llamar "crossmedia" de manera general cuando se quiere enfatizar la idea de utilizar el mismo contenido adaptado a diferentes medios y "transmedia" cuando se insiste en la posibilidad de que el consumidor experimente contenidos que son complementarios a través de diferentes medios. Se trata, por decirlo así, de sumergir al consumidor en el contenido -un paradigma típico de los videojuegos- utilizando para ello las características únicas de cada formato y cada soporte, pero haciéndolo de manera coordinada.

A modo de resumen final surgen dos consecuencias inmediatas de este reto. En primer lugar, la necesidad de realizar la producción de contenidos de manera que cubra diferentes medios, diferentes plataformas, diferentes formatos y diferentes soportes. Este fenómeno ya es patente en subsectores como el de los contenidos móviles. Desde el punto de vista de la producción, además de cuestiones técnicas y económicas, obligará a pensar en los diversos ámbitos que componen la industria de los contenidos como sectores no aislados, produciendo una convergencia entre los mismos. Esta convergencia terminará trasladándose también a los formatos, los soportes y reforzando las preferencias de los usuarios sobre la facilidad de uso transmediático. Precisamente, ésta es la segunda -y quizá más importante- consecuencia: nuestro viaje a través de nuevos formatos y sopor- 
tes para los contenidos digitales no ha terminado, de hecho tan solo acaba de comenzar.

\section{Notas}

1. Los datos que figuran en este artículo están extraídos de un próximo informe sobre los contenidos digitales publicado por el Parlamento Europeo.

Feijóo, Claudio; Lindmark, Sven et al. (2013, forthcoming) "Public and commercial modes of access to content and information in the digital era". Culture and Education. IP/B/ CULT/IC/2012-018. European Parliament. Brussels.

Para más detalles sobre el mismo, contactar con el autor.

2. Un nombre, el de industrias culturales, que agruparía al conjunto heterogéneo de los sectores de la música, el cine, la televisión, los videojuegos, el ámbito editorial (libros, revistas, periódicos), otros servicios de información (agencias de noticias, portales, directorios, etc), pero también diversas actividades con participación pública como museos, bibliotecas, patrimonio, etc. En el ámbito anglosajón ha ganado auge recientemente la expresión "media and content industries", aquí líbremente traducida como industria de los contenidos.

3. La producción a gran escala permite producir objetos a menor precio.

4. En español llamamos "soportes" a todo lo que sustenta o lleva la información o los datos: papel, formato electrónico o digital (pdf, doc, jpg...), online, offline, televisión, teléfonos inteligentes..., pero no existe una palabra equivalente en inglés. En este idioma -además de con los nombres de los soportes concretos- se puede expresar mediante devices, consumer/user equipment, media, platforms...

5. Productores-consumidores.

6. Time shifting, cambio de tiempo o pausa, grabación de un programa (TV, radio) para su posterior visionado o escucha en un momento más adecuado para el usuario.

7. Aunque con algunas notorias excepciones en productos de nicho, como puede ser el caso de las enciclopedias.

8. EUA, Canadá, UE-5, Japón.

9. Acceso gratuito pero pago para el uso de ciertas funciones (hemeroteca...) o ciertos contenidos (información financiera...).

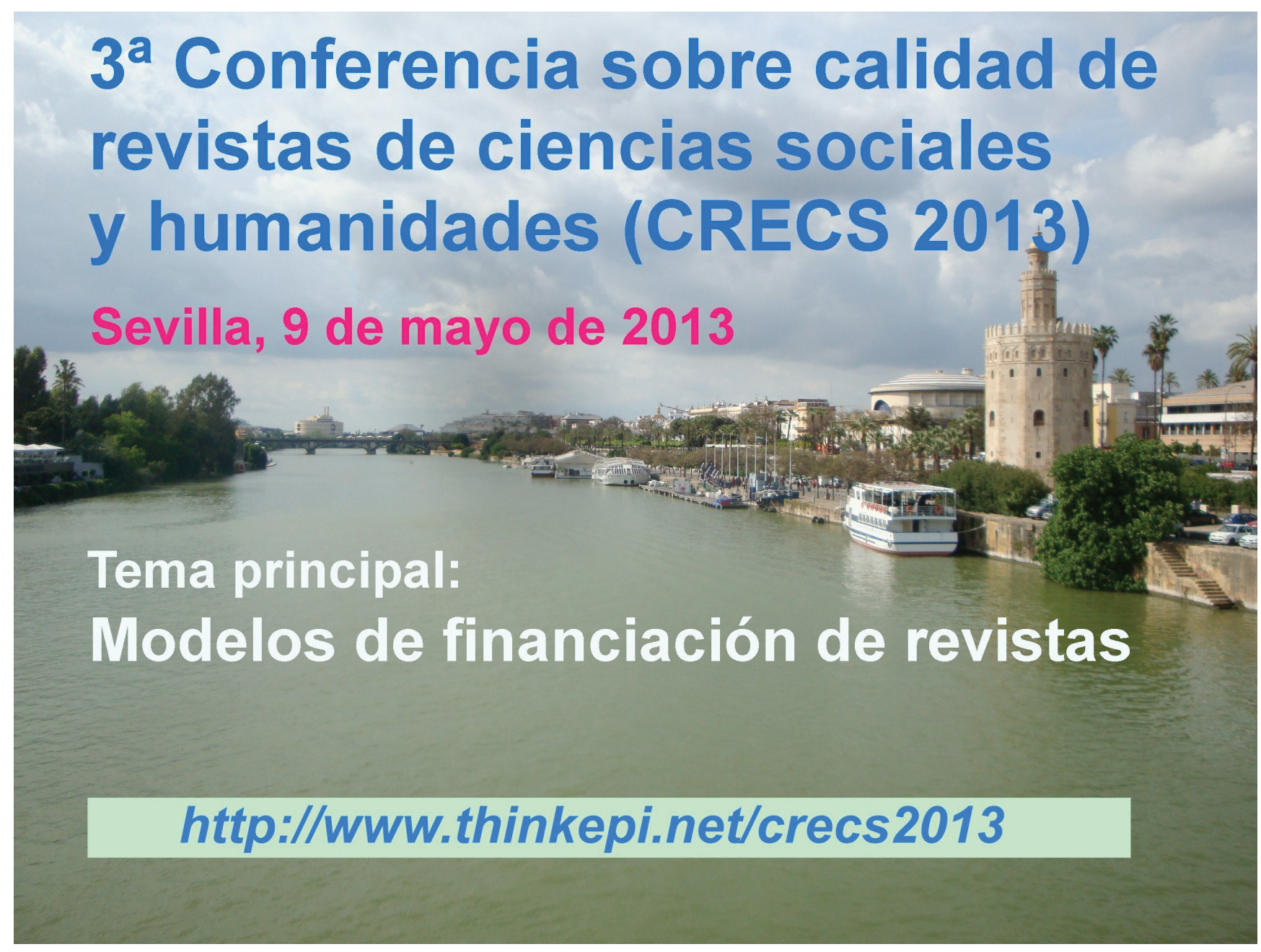

